\title{
Benign fibrous histiocytoma of lung in a child: The diagnostic dilemma
}

\author{
Vaibhav Pandey ${ }^{1}$, AN Gangopadhyay ${ }^{2}$, DK Gupta $^{3}$, Mohan $_{\text {Kumar }}{ }^{4}$ \\ ${ }^{I}$ M.B.B.S, M.S, M.Ch., Senior Resident, Department of Paediatric Surgery, Institute of Medical Sciences, \\ Banaras Hindu University, Varanasi, U.P. \\ ${ }^{2}$ M.B.B.S, M.S, M.Ch., Professor Department of Paediatric surgery, Institute of Medical Sciences, Banaras \\ Hindu University, Varanasi, U.P. \\ ${ }^{3}$ M.B.B.S, M.S, M.Ch., Professor Department of Paediatric surgery, Institute of Medical Sciences, Banaras \\ Hindu University, Varanasi, U.P. \\ ${ }^{4}$ M.B.B.S, M.D. Professor Department of Pathology, Institute of Medical Sciences, Banaras Hindu University, \\ Varanasi, U.P.
}

\begin{abstract}
Benign fibrous histiocytoma $(B F H)$ is an extremely rare mediastinal tumor with few cases reported in 20-40 year age group [1,2]. We herein report first case of paediatric BFH of lung. The diagnostic and therapeutic implications of the tumor should be in the armamentarium of the treating surgeon, as it will reduce pitfalls in management of this tumor owing to its rarity, benign nature and excellent prognosis.
\end{abstract}

Keywords: "BFH", "Fibrous histiocytoma lung", "Paediatric lung tumour",

\section{Introduction}

Benign fibrous histiocytoma (BFH) is a rare tumors of mixed origin, i.e., fibroblastic and histiocytic with unknown etiology [1]. Lower extremity, head and neck region are the most common sites of presentation [3]. BFH of lung is very rare, presenting mostly in 20-40 year age group [2]. We herein report the first case of mediastinal BFH in paediatric age group with difficult diagnosis and excellent prognosis.

\section{Case}

A 4 year child presented to us with the complaints of recurrent episodes of fever, non-productive cough and dyspnea for duration of 3 months. He was otherwise healthy and asymptomatic. For above complaints he was managed as a case of pneumonia outside. On physical examination, child was tachypneic with respiratory rate 34/min, right side chest movement was restricted and breath sounds were absent in the upper part. Rest of systemic examination was unremarkable. Roentgenograms of the chest showed a space occupying lesion in the left upper lobe [Fig 1]. Computer tomography of chest showed heterogeneously enhancing mass in the left lung field $6.5 \times 8.5 \mathrm{~cm}$ in size, with possibility of germ cell tumour or neuroblastoma [Fig 2]. Alpha-fetoprotein was $2 \mathrm{ng} / \mathrm{mL}$ and 24 hour urinary vanillylmandelic acid was $3 \mathrm{mg} / \mathrm{g}$ of creatnine. The good general condition of child without any metastasis and resectable nature of lesion prompted us for thoracic exploration. A left sided thoracotomy was performed, which showed a well-encapsulated, soft tissue mass arising from upper lobe of left lung [Fig 3]. Histopathology showed a biphasic pattern of histiocytes and fibroblasts and undifferentiated mesenchymal cells with characteristic hyalinization similar to the fibro histiocytic neoplasm [Fig 4]. Tumour was CD68 positive on Immunohistochemistry A diagnosis of exclusion was made on the basis of tumor location, radiographic and microscopic characteristics, other benign lesions can be eliminated. Post operative course was uneventful. No post operative radiotherapy or chemotherapy was given in post operative period. The child is normal at three year follow up.

\section{Discussion:}

Benign fibrous histiocytoma (BFH) is a rare tumour of unknown etiology and is most commonly found in women in the third or fourth decades [5]. Lower extremity, head and neck region are the most common sites of presentation [3]. Benign fibrous histiocytoma in the posterior mediastinum and lung has been previously reported, mostly among females in 20 and 40 years [4, 7]. In our case preoperative diagnosis of mediastinal tumour was made on basis of CT scan. Intraoperatively we found tumor to be arising from and completely confined to lung. BFH are well circumscribed and pseudo-encapsulated, with central hemorrhage or cystic changes being an occasional feature with presence of characteristics biphasic pattern of histiocytes and fibroblasts with myofibroblasts and undifferentiated mesenchymal cells $[2,6]$. Occasionally, focal areas of necrosis are seen [7]. In our case the tumour was well encapsulated and showed the characteristics biphasic pattern on histopathology. BFH involving deeper body parts are generally larger and better circumscribed than their cutaneous counterparts. The diagnosis of BFH is supported by CD 68 positivity on Immunohistochemistry 
[8]. Nearly half of these tumors are $5 \mathrm{~cm}$ or greater when excised compared with the majority of cutaneous fibrous histiocytoma, which are $<3 \mathrm{~cm}$ [1]. The benign nature of lesion warrants cure with surgical excision only with excellent prognosis $[5,6]$. So no radiotherapy or chemotherapy was given in postoperative period.

\section{Conclusion}

The diagnosis of BFH of lung is of exclusion. The diagnostic and therapeutic implications of the tumor should be in the armamentarium of the treating surgeon, as it will reduce pitfalls in management of this tumor owing to its rarity, benign nature and excellent prognosis.

Legends:

Fig1. X-ray chest showing space occupying lesion left lung

Fig2. Computer tomography of Left lung showing mediastinum based heterogeneously enhancing mass

Fig3. 6.5 X $8.5 \mathrm{~cm}$ in size well encapsulated mass from left lung.

Fig4. X 400 Hitiocytes with a vesicular nulei, spindle cells with intervening collagen and lymphoctes

\section{References}

[1] Enzinger, Weiss. Soft tissue tumor, 4th ed. Mosby; 2001. p. 441-55.

[2] Sajjad SM, Begin LR, Dail DH, Lukeman JM. Fibrous histiocytoma of lung-a clinicopathological study of two cases. Histopathology 198;5(3):325-34.

[3] Fletcher CD. Benign fibrous histiocytoma of subcutaneous and deep soft tissue: clinicopathologic analysis of 21 cases. Am J Surg Pathol 1990;14:801-809.

[4] Sood N, Singhal VS. Benign fibrous histiocytoma of the mediastinum. Indian J Chest Dis Allied Sci 1992;34:33-7

[5] Lago JV, Pujol JL, Reboiras SD, Larrauri J, Schacke M. Fibrous histiocytoma of the lung. Thorax 1976;31:475-479.

[6] van Giffen NH, van Rhijn LW, van Ooij A, Cornips E, Robben SG, Vermeulen A, Maza E. Benign fibrous histiocytoma of the posterior arch of $\mathrm{C} 1$ in a 6-year-old boy: a case report. Spine 2003;28(18):E359-63.

[7] Fletcher CD. Diagnostic histopathology of tumors. Churchill Livingstone; 2000. p. 1014-505.

[8] Prieto VG, Reed JA, Shea CR. Immunohistochemistry of dermatofibromas and benign fibrous histiocytomas. J Cutan Pathol 1995;22(4):336-41.

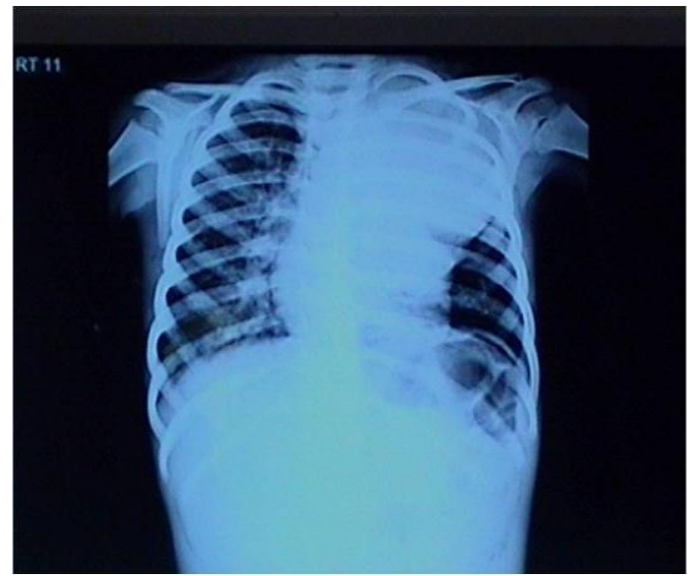

Fig 1

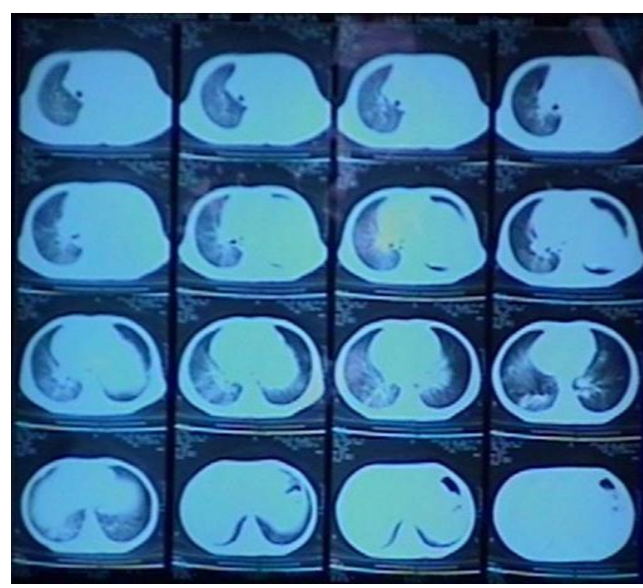

Fig. 2 


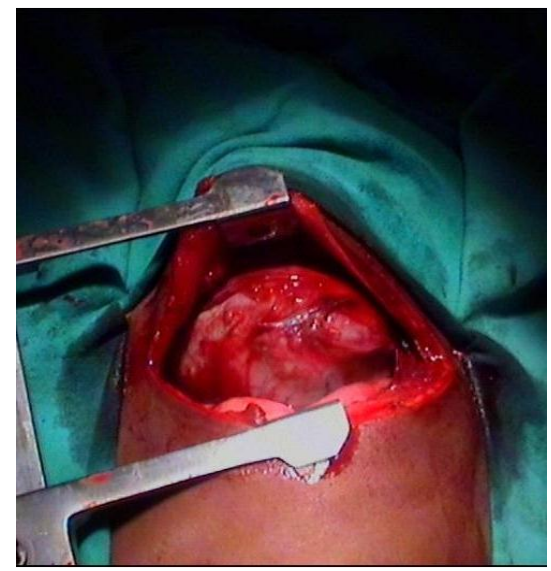

Fig 3

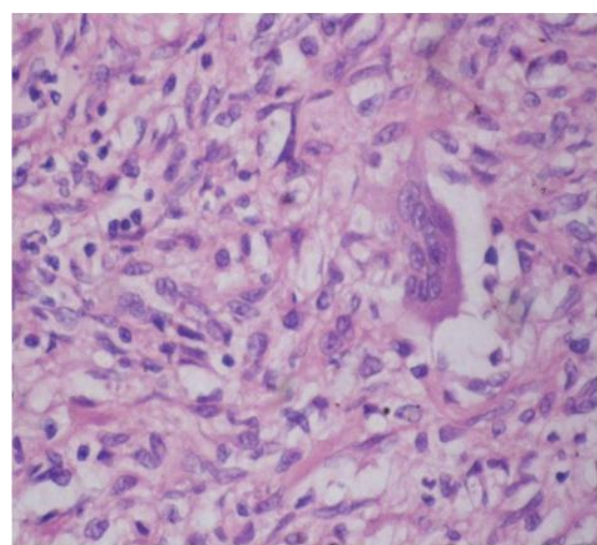

Fig 4 